\title{
Pancreas Divisum
}

\author{
Matthew J. DiMagno, MD and Erik-Jan Wamsteker, MD \\ University of Michigan Medical School, Division of Gastroenterology and Hepatology, Department \\ of Internal Medicine, Ann Arbor, MI 48109, USA
}

\section{Abstract}

We review important new clinical observations in pancreas divisum (PD) made since 2007. PD is common and has the same prevalence in the general population and idiopathic pancreatitis (IP). Up to $53 \%$ of patients with PD and IP have underlying idiopathic chronic pancreatitis (CP), and in rigorous prospective clinical follow-up and/or natural history studies, many with idiopathic recurrent acute pancreatitis (IRAP) have idiopathic CP. According to retrospective studies, PD does not modify the natural course of nonalcoholic or alcoholic CP. CFTR and/or SPINK1 gene mutations associate with IP (idiopathic CP and IRAP) independently of the presence of PD. More than one third of patients with pancreatitis or presumed pancreaticobiliary pain respond to placebo. Authors of uncontrolled studies report a significant symptomatic response to surgery and endotherapy in patients with IP and PD, but the response remains unproven and is largely limited to those with IRAP and not idiopathic CP or chronic pain.

\section{Keywords}

Pancreas divisum; Acute pancreatitis; Chronic pancreatitis; Sphincterotomy

\section{Introduction}

Pancreas divisum (PD) is the most common congenital variation of pancreatic duct anatomy, arising when the embryological ventral and dorsal endodermal buds fail to fuse ("classic" PD) or only partially fuse ("incomplete" PD). With this ductal variant, pancreatic drainage is mainly through the accessory papilla. The possibility that PD has pathophysiological consequences related to idiopathic pancreatitis (IP) emerged in the 1970s, when endoscopists identified PD by endoscopic retrograde cholangiopancreatography (ERCP) in patients. Currently, it remains controversial whether PD causes IP and whether surgical or endoscopic drainage procedures of the duct of Santorini reduce pain and attacks of pancreatitis [1•]. In this review, we discuss terminology for IP, the hypothesis that PD causes IP, the prevalence of PD, alternative explanations for IP in patients with PD, and the results of treatment and response to placebo.

Corresponding author: Matthew J. DiMagno, MD, Department of Internal Medicine, Division of Gastroenterology and Hepatology, University of Michigan Medical School, 1150 West Medical Center Drive, Room 6520 MSRB I, Ann Arbor, MI 48109-0682, USA., mdimagno@umich.edu, Telephone number: (734) 763-7278, Fax number: (734) 763-2535.

Conflicts of interest: M. DiMagno—none; E-J. Wamsteker-none.

Disclosure

Dr. DiMagno is supported by NIH grants K08 DK073298 and R21 AA017271. 


\section{Terminology for Pancreatitis}

Authors use different terminology to describe "recurrent pancreatitis." The most commonly used term is idiopathic recurrent acute pancreatitis (IRAP), which sidesteps the consideration that IRAP patients have chronic pancreatitis (CP) from the initial presentation of symptoms. The initial presentation of patients with early-onset idiopathic CP is pain, followed by recurrent attacks of pain at variable intervals of months to years, and many years later the hallmark features of CP (calcification, diabetes, and malabsorption) occur [2,3]. Many patients with PD and IRAP have CP. DiMagno and DiMagno [1 $]$ analyzed data from 41 endoscopic studies and reported that up to 53\% of patients had evidence of CP. To avoid confusion in this review article, we use the term IP and include within this term the diagnoses of acute and chronic IP made by others. The term recurrent acute pancreatitis (RAP) describes all patients with this condition, regardless of etiology, most of whom have identifiable causes of RAP (eg, biliary lithiasis, hypertriglyceridemia, hypercalcemia). The term idiopathic RAP (IRAP) indicates patients who have no identifiable cause of RAP, who, in our opinion, have early idiopathic CP.

\section{Hypothesis}

The hypothesis that obstruction of the minor papilla causes pancreatitis serves as the basis for performing drainage procedures of the duct of Santorini in patients with IP and PD [1•]. If the hypothesis is correct, we should expect:

1. The prevalence of PD should be greater in IP than the general population.

2. A dilatated dorsal duct system should be present if there is a functionally significant obstruction of the dorsal duct.

3. Pathological changes should only develop in the dorsal duct.

4. The frequency or severity of recurrent attacks of IP should diminish with drainage procedures of the duct of Santorini.

\section{Prevalence of PD}

For more than 150 years, anatomists and later clinicians used different methods to describe PD, including autopsy, surgery, endoscopic retrograde pancreatography (ERCP), magnetic resonance pancreatography (MRCP) and secretin-MRCP (S-MRCP). The aim of more than $90 \%$ of autopsy studies was to classify normal variations of human pancreatic ducts and structures, whereas the aim of ERCP studies was to determine the relationship between PD and IP.

Accumulating data indicate that the prevalence of PD is no greater in IP compared to the general population. Initially, investigators using ERCP reported a greater prevalence of PD in IP compared to the general population [4-6]. These data later were contradicted by other ERCP studies [7-9]. Multiple factors might account for these differences; the early studies [4-6] might have been biased by referral pattern, patient selection, and inclusion of subjects with poorly characterized pancreatic disease (see "Terminology for Pancreatitis").

DiMagno and DiMagno [1•] recently reported a comprehensive analysis of primary sources from 23 autopsy studies, $41 \mathrm{ERCP}$ studies, and $13 \mathrm{MRCP}$ studies to determine the prevalence of PD in IP compared to the general population and in idiopathic "acute pancreatitis" compared to CP. Patients were categorized as having no pancreatitis, IP, and acute pancreatitis and $\mathrm{CP}$ when sufficient detail supported this designation. In the autopsy and MRCP studies, the prevalence of PD in the general population was about 8\% (Fig. 1A). In the ERCP studies, however, the prevalence of PD was about $4 \%$ in the general population 
and about $8 \%$ in patients with IP (Fig 1A). These data support the conclusion that in ERCP studies, the prevalence of PD in the general population is because of under recognition and/ or referral bias, and that there is no association between PD and IP.

Controversy about this conclusion exists. For example, analysis of six of the 41 ERCP studies and two of the seven S-MRCP studies that classified IP patients as acute pancreatitis or $\mathrm{CP}$ shows a prevalence of $\mathrm{PD}$ that was significantly greater in acute pancreatitis compared to CP in ERCP but not in S-MRCP studies (12.0 vs 6.8) [1•]. The validity of this finding is questionable, however, because the increased PD prevalence in acute pancreatitis was directly attributable to two publications from the same investigators $[5,10]$. There was no increased prevalence of PD in acute pancreatitis compared to autopsy in most studies, and the authors of the largest ERCP study of 304 patients with PD reported a similar prevalence of PD in acute pancreatitis and CP groups (7.5\% vs 6.4\%) [8]. To explain the contrasting observations among studies, it is noteworthy that a dorsal duct pancreatogram was performed $50 \%$ less frequently in studies reporting an increased prevalence of PD in acute pancreatitis compared to the study by Delhaye et al. [8], indicating that a diligent search for PD (by examining both ductal systems) was not always performed in patients without a pancreatitis history. Hence, we interpret these data as indicating that PD is no more prevalent in idiopathic acute pancreatitis compared to CP.

More recently, a systematic review of ERCP detection rates for PD [11•] included 17 studies: six from Asia, three from Europe, and eight from the United States. Overall, endoscopists detected PD in $2.9 \%$ of ERCPs ( 899 of 31413 ), but prevalence varied significantly by geographic location: $1.5 \%$ (317 of 21636 ERCPs) in Asia, significantly lower than 5.7\% (395 of 6578; $P<0.001$ ) in the United States and 6.0\% (899 of 31413; $P<$ $0.001 \%$ ) for Europe. The US and European data approximate data of the 41 ERCP studies analyzed by DiMagno and DiMagno [1•], who reported a PD prevalence of $4.1 \%$ of 16,078 subjects without IP. Perhaps the most interesting observation by Liao et al. [11•] is that the prevalence of PD appears to vary by different geographic locations. These regional differences, however, are likely attributable to the degree that endoscopists search for PD (by examining both ductal systems) or to referral bias or other factors as discussed previously.

\section{Natural History}

Although we do not subscribe to the hypothesis that PD underlies IRAP [4], if this hypothesis were true, $\mathrm{PD}$ may also underlie $\mathrm{CP}$. The rationale for the latter hypothesis is that up to $53 \%$ of patients enrolled in studies with IRAP had evidence of $\mathrm{CP}[1 \bullet]$ and that RAP (particularly alcoholic) evolves into CP [12] by a series of necrotic and fibrotic events, as originally proposed in 1946 by Comfort et al. [13]. The studies of Barthet et al. [14] and Spicak et al. [15•], however, do not support the hypothesis that PD is involved in the pathogenesis of PD.

Barthet et al. [14] reported that the clinical course and morphological features of 40 patients with calcifying CP associated with heavy alcohol use were not modified by PD. Twenty had PD and 20 had a normally fused pancreas. The only significant difference in clinical characteristics was that pancreatic calcifications were less common in the PD group (8 vs $14 ; P=0.05)$. Cambridge classification was similar among groups, but the PD group had minor differences of uncertain significance, including less frequent irregularity of the main pancreatic duct and more frequent dilation of side branches. Further evidence that PD was an incidental finding is that isolated dorsal segment disease occurred in only $45 \%$ of patients with PD. The lower than expected frequency of isolated dorsal duct involvement was 
corroborated by Spicak et al. [15•] (see below) and by the pooled analysis of DiMagno and DiMagno [1•].

Spicak et al. [15•] recently reinvestigated whether PD associated with an altered natural history of CP by evaluating 87 patients with CP, 30 with PD and 57 without PD. Similar to the study by Barthet et al. [14], most patients in both groups had heavy alcohol use and there were no significant differences among gender, age at onset of disease, clinical presentation, course of the disease, and frequency of complications. Additional data that do not support PD predisposing to pancreatitis by obstructing the dorsal duct are that most patients had disease involving the ventral segment whether PD was present or not (75\% vs $72 \%$ ), and that isolated dorsal segment disease was less frequent than expected and occurred nearly equally in the alcoholic versus nonalcoholic groups (25\% vs $28 \%)$. These clinical and anatomic data indicate that PD does not modify the natural course of chronic nonalcoholic or alcoholic pancreatitis and that PD does not play a dominant role in the pathogenesis of $\mathrm{CP}$, if it plays a role at all.

Although prevalence data (Fig. 1B), natural history studies, and the results of endoscopic treatment studies (see section on therapy) do not support a meaningful association between $\mathrm{PD}$ and $\mathrm{CP}$, it is important to recognize if a subset of IRAP patients with or without PD present as, or evolve into, CP. In $10 \%$ to $20 \%$ of persons with a first attack of acute pancreatitis, no cause is identified after completing a routine clinical evaluation, biochemical tests, transabdominal ultrasound, and/or CT [16]. Of these with a first attack of IP, more intensive evaluation with endoscopic ultrasound (EUS) may identify a cause in about $80 \%$ [17]. Fewer than $15 \%$ of patients with a first attack of acute pancreatitis develop RAP [18], and far fewer develop IRAP (no identifiable cause of RAP). Few studies combine complete diagnostic testing with prospective follow-up, but available data indicate that most patients labeled as having IRAP eventually will have an identifiable cause of RAP, including idiopathic CP, genetic mutations (see "Association of IP with Genetic Mutations and PD"), biliary disease (gallbladder microlithiasis, choledocholithiasis, biliary sludge), or unusual lesions such as ampullary lesions or pancreatic cancer.

Three long-term studies followed patients with IRAP prospectively for about 18 to 36 months and showed that the majority had RAP due to an identifiable cause, more commonly idiopathic CP (26\%-50\%) rather than a biliary cause [19-21]. An interesting finding in one study was that pancreatic duct stenting compared to no stenting had no effect on pancreatictype pain (32\% vs $40 \%$ ) or the development of findings of idiopathic CP (27\% vs $26 \%$ ) [20]. Hence, idiopathic CP is a common etiology of RAP [19-21], it is likely that early- and lateonset idiopathic CP develop as a consequence of RAP because of unrecognized etiologies (eg, genetic abnormalities or environmental factors) and that pancreatic duct stenting does not prevent the course of idiopathic CP [20].

\section{Association of IP with Genetic Mutations and PD}

IP associates with mutations in primarily three genes. Mutations in the cationic trypsinogen (PRSS1) gene occur primarily in hereditary pancreatitis [22] and less commonly in IP [23]. More common gene mutations associated with IP involve the CFTR $[24,25]$ and/or SPINK1 gene [26], the latter commonly occurring in tropical calcific CP [27]. Up to 50\% of patients with early-onset idiopathic CP have mutations of the CFTR and/or SPINK1 genes [28-30]. Also, patients with IRAP commonly have CFTR mutations (up to 38\%) [30,31] or SPINKI mutations (about 11\%) [32]. As previously discussed (see "Terminology for Pancreatitis"), these patients likely have idiopathic CP [33], a premise supported by findings that CFTR and SPINK1 gene mutations are not associated with single episodes of human acute pancreatitis [32,34]. 
CFTR mutations may confer increased susceptibility to IP by at least two mechanisms: pancreatic ductal plugging and obstruction by viscous, proteinaceous ductal secretions [35] and by sensitizing the pancreas to an exuberant inflammatory response to injury [36,37]. SPINK1 mutations may predispose to IP by disrupting the capacity of pancreatic acinar cells to limit trypsin activity when premature activation occurs intracellularly. It is tantalizing to consider that other patients with IP have a less common gene mutation (eg, chymotrypsinogen $\mathrm{C}$ gene [38] or calcium-sensing receptor gene [39,40]), a yet to be discovered gene mutation, or a nongenetic alteration in protein function, a premise based on findings that patients may have a variant CF phenotype without CFTR gene mutations [41,42], including those with CP from known causes [43].

Patients with IP, with or without PD, have a similar prevalence of CFTR and/or SPINK1 gene mutations. Investigators from the University of Indiana [31], who used a limited screening test, reported that in a Caucasian population, the frequency of CFTR mutations was higher in patients with PD and IP compared to PD without IP (22\% vs $0 \%)$. The true prevalence of CFTR mutations in IP and PD is likely higher because the frequency of CFTR mutations in IP is greater when investigators use an exhaustive genetic screening test $[24,25,30]$. Furthermore, even when genetic testing identifies no CFTR mutations, CFTR functional impairments are common in patients with IP and PD. Gelrud et al. [44] studied 12 patients with IP and PD and found that nasal potential difference, an indicator of CFTR ion channel function, was intermediate between those observed for healthy controls and classic CF patients. In addition, Gelrud's study implies that IP with PD is uncommon (only 12 patients found at three institutions) and that in this uncontrolled study, patients who have CFTR ion channel dysfunction generally do not respond to endoscopic therapy; only two of 12 patients who underwent endoscopic or surgical interventions had resolution of symptoms, even though all had multiple ERCPs (range 2-5), 10 had either pancreatic stent and/or sphincterotomy, and eight had cholecystectomy.

Recently, investigators from New Delhi, India, found the frequency of SPINK1 mutations was increased but similar in three pancreatitis groups: $41.6 \%$ of IP with PD (5 of 12), 43.3\% of CP (13 of 30), and $35.7 \%$ of RAP without PD (5 of 14) compared to $2 \%$ of healthy controls (1 of 50) [45]. No patients had CFTR mutations but frequencies of CFTR polymorphisms were increased and similar in the two pancreatitis groups: $25 \%$ of IP and PD ( 3 of 12 ) and $33.3 \%$ of CP (10 of 30) compared to $0 \%$ of healthy controls ( 0 of 50 ). The authors claim that relative obstruction at the minor papilla owing to PD plays a definite role in the pathogenesis of pancreatitis because endoscopic therapy in eight of 12 patients with PD had a 50\% reduction per year in the frequency of attacks of pancreatitis, including three of five with SPINK1 mutations. This response contrasts with the negative endoscopic outcomes reported by Gelrud et al. [44], but may be explained by the short duration of follow-up, lack of treatment controls, and possibly the involvement of different gene mutations.

Findings that CFTR and/or SPINK1 gene mutations have a similar prevalence in IP with and without PD indicates that the gene mutation is the common underlying factor for causing pancreatic inflammation and that PD is likely an incidental finding in the setting of IP. CFTR and SPINK1 mutations likely have synergistic effects on the susceptibility to IP in patients with PD, similar to that observed in IP [28], possibly because different molecular mechanisms are affected. Data are insufficient, however, to completely exclude the possibility that in some patients PD participates with CFTR and/or SPINK1 mutations as a "two-hit" phenomenon that increases the susceptibility to IP. 


\section{Placebo Response Rate}

When either interventional or medical treatment is tested against placebo, patients with pancreatitis or presumed pancreaticobiliary pain have a high placebo response rate. For example, in patients with type 2 and 3 sphincter of Oddi dysfunction, Wilcox [46] reported a $38 \%$ endoscopic placebo response rate. In patients with abdominal pain and CP, others reported a similar 35\% to 36\% placebo response rate in trials of octreotide versus placebo [47] or the cholecystokinin-A receptor antagonist loxiglumide versus placebo [48]. Also, patients with $\mathrm{CP}$ have a dramatic, but less quantifiable, placebo response in a trial of antioxidants versus placebo [49]. These $35 \%$ to $38 \%$ placebo response rates are strikingly similar to the $32 \%$ "therapeutic response" of endoscopic drainage of the pancreatic duct in a randomized controlled trial of patients with large-duct CP [50]. Recognition of the placebo response led Cooperman et al. [51] to comment that it remains unclear whether a perceived therapeutic response to dorsal duct drainage procedures in patients with PD represents "...a satisfactory result, stabilization of the disease, symbiosis between patient and symptoms, or fear of admitting persistent symptoms...." Hence, only properly randomized and controlled trials of treatment versus no treatment will determine whether endoscopic therapy is effective for reducing pain in patients with PD.

\section{Endoscopic and Surgical Therapy for IP and PD}

A two-part clinical controversy published in 2007 [1•] outlined major limitations of studies of endoscopic and/or surgical therapy of patients with IP and PD. Specifically, studies were small, largely retrospective, lacked a control group (to assess a placebo response rate), and had limited long-term follow-up. Therefore, the indications for and the true response to minor papillotomy remain unclear in clinical practice. Two new studies [11•,52•], an editorial [53], and a recent review article [54] highlight these issues.

In a recent, retrospective study, Chacko et al. [52•] reported that the minor papilla endotherapy (MPE) response rate or clinical improvement was 76\% for IRAP but much lower for $\mathrm{CP}(42 \%)$ and chronic abdominal pain (33\%). The authors defined improvement as a $\geq 50 \%$ reduction in any endpoint for IRAP, $\mathrm{CP}$ or chronic abdominal pain. Specific endpoints for IRAP included annual episodes of acute pancreatitis, emergency department visits or hospitalizations. Endpoints for $\mathrm{CP}$ and chronic abdominal pain were pain scores, use of narcotic analgesics, annual emergency department visits or hospitalizations. The authors concluded that MPE is a reasonable first step in the absence of chronic pain. An important limitation acknowledged by the authors is that short-term follow-up may be inadequate to assess response in some patients with IRAP, who have long, pain-free intervals. A further challenge in assessing the response rate in patients with IRAP is that some develop chronic pain. Multiple additional factors limit the generalizability of this study: the investigators included patients with incomplete PD and previous endotherapy, some patients classified as having IRAP had evidence of CP, follow-up was short, endpoints were heterogeneous, and there was no intention-to-treat analysis. An editorial [53] concluded that patients with IRAP represent the subset of patients most likely to have clinical benefit from MPE, but that further studies are needed to evaluate the long-term benefit from MPE, preferably designed as randomized, controlled studies in which details of disease presentation and pre-procedural natural history are documented.

Liao et al. [11•] reported in a systematic review that response to endotherapy and surgery in patients with PD depends on whether patients have IRAP, CP, or chronic abdominal pain. The greatest response rates, defined broadly as the percentage of patients with complete, partial, or overall pain relief at follow-up, occurred in IRAP (79.2\%-83.2\%) but significantly less in CP $(69.0 \%-66.7 \%)$ and chronic abdominal pain $(54.4 \%-51.6 \%)$. This 
analysis of uncontrolled studies supports the findings of multiple previous studies that either the endoscopic or surgical papillary therapy is "effective." Unfortunately, as described above, these studies lack proper controls and randomization, outcome measures remain too broadly defined, and follow-up is limited. These limitations apply mostly to IRAP patients, who are purported to benefit greatest from minor papillary therapy.

Finally, Delhaye et al. [54] stressed that the diagnosis of PD can readily be made noninvasively with secretin MRCP imaging. Further, they state that PD as a cause of IP remains controversial for several reasons, including the inability to demonstrate an increased incidence of PD in IP compared with control patients, and that recruitment bias may have led to an overestimation of the prevalence of PD in other ERCP studies examining IRAP and PD. They suggest, however, that there may be a subset of patients with PD who may benefit from minor papillotomy, based on S-MRCP imaging results of delayed duodenal drainage, even though secretin-induced duct dilatation detected by US/MRCP is abnormal in 50\% of normal controls [55]. Whether this result, suggesting obstruction at the minor papilla, may allow for proper selection for minor papillary therapy, awaits prospective studies demonstrating a beneficial outcome.

\section{Conclusions}

Is PD “....an unrecognized cause of many cases of recurrent acute pancreatitis”? [56]. No! $\mathrm{PD}$ is as common in the general population as in IP. Rigorous prospective clinical follow-up and/or natural history studies indicate that many with IRAP have idiopathic CP. Indeed, up to 53\% of patients with PD and IP included in studies have underlying idiopathic CP. In retrospective studies, PD does not modify the natural course of nonalcoholic or alcoholic CP. CFTR and/or SPINK1 gene mutations associate with IP (idiopathic CP and IRAP), and the association is unrelated to the presence of $\mathrm{PD}$, indicating that $\mathrm{PD}$ is likely an incidental finding in IP and that gene mutations are common underlying factors responsible for pancreatic inflammation. Data are insufficient, however, to completely exclude the possibility that, in some patients, PD combines with CFTR mutations as a "two-hit" phenomenon that increases the susceptibility to IP. More than one third of patients with pancreatitis or presumed pancreaticobiliary pain respond to placebo. Predicting endoscopic or surgical response is not standardized. In uncontrolled studies, authors report a significant symptomatic response to surgery and endotherapy in IP patients with PD, but the response is largely limited to IRAP and not idiopathic $\mathrm{CP}$ or chronic pain. Endoscopic therapy of patients with IP and PD without evidence of CP remains unproven. This controversy can be settled by randomized controlled trials of endoscopic therapy versus sham intervention with long-term follow-up.

\section{Abbreviations}

$\begin{array}{ll}\text { AP } & \text { Acute pancreatitis } \\ \text { PRSS1 } & \text { cationic trypsinogen } \\ \text { CCK } & \text { cholecystokinin } \\ \text { CP } & \text { chronic pancreatitis } \\ \text { CI } & \text { confidence interval } \\ \text { CFTR } & \text { cystic fibrosis transmembrane conductance regulator } \\ \text { EUS } & \text { endoscopic ultrasonography } \\ \text { ERCP } & \text { endoscopic retrograde cholangiopancreatography }\end{array}$




$\begin{array}{ll}\text { IP } & \text { idiopathic pancreatitis } \\ \text { IRAP } & \text { idiopathic recurrent acute pancreatitis } \\ \text { MRCP } & \text { magnetic resonance cholangiopancreatography } \\ \text { MPD } & \text { main pancreatic duct } \\ \text { MPE } & \text { minor papilla endotherapy } \\ \text { PD } & \text { pancreas divisum } \\ \text { PFT } & \text { pancreatic function test } \\ \text { RCT } & \text { randomized controlled trial } \\ \text { RAP } & \text { recurrent acute pancreatitis } \\ \text { S-MRCP } & \text { secretin-magnetic resonance cholangiopancreatography } \\ \text { SPINK1 } & \text { serine protease inhibitor, Kazal type 1 } \\ \text { SOD } & \text { sphincter of Oddi dysfunction }\end{array}$

\section{References and Recommended Reading}

Papers of particular interest, published recently, have been highlighted as:

- Of importance

1•. Fogel EL, Toth TG, Lehman GA, et al. Does endoscopic therapy favorably affect the outcome of patients who have recurrent acute pancreatitis and pancreas divisum? Pancreas. 2007; 34(1):2145. To address an important and long-standing controversy in clinical pancreatology, this twopart discussion focuses on data and arguments for and against endoscopic therapy for patients with recurrent acute pancreatitis and PD. Coauthors Fogel, Toth, and Lehman report "The Case for Endoscopic Therapy" and coauthors DiMagno and DiMagno report "The Cases Against Endoscopic Therapy." The section by DiMagno and DiMagno also includes multiple new analyses of the world's literature to report prevalence data of PD in idiopathic pancreatitis based on whether data were derived from autopsy, endoscopic, or MRCP literature. [PubMed: 17198181]

2. Layer P, Yamamoto H, Kalthoff L, et al. The different courses of early- and late-onset idiopathic and alcoholic chronic pancreatitis. Gastroenterology. 1994; 107(5):1481-1487. [PubMed: 7926511]

3. DiMagno, MJ.; Wamsteker, EJ.; Lee, A. Chronic Pancreatitis. BMA House; London: 2009. https://online.epocrates.com/u/291167/Chronic+pancreatitis

4. Cotton PB. Congenital anomaly of pancreas divisum as cause of obstructive pain and pancreatitis. Gut. 1980; 21(2):105-114. [PubMed: 7380331]

5. Sahel J, Cros RC, Bourry J, Sarles H. Clinico-pathological conditions associated with pancreas divisum. Digestion. 1982; 23(1):1-8. [PubMed: 7084565]

6. Richter JM, Schapiro RH, Mulley AG, Warshaw AL. Association of pancreas divisum and pancreatitis, and its treatment by sphincteroplasty of the accessory ampulla. Gastroenterology. 1981; 81(6):1104-1110. [PubMed: 7286588]

7. Burtin P, Person B, Charneau J, Boyer J. Pancreas divisum and pancreatitis: a coincidental association? Endoscopy. 1991; 23(2):55-58. [PubMed: 2050007]

8. Delhaye M, Engelholm L, Cremer M. Pancreas divisum: congenital anatomic variant or anomaly? Contribution of endoscopic retrograde dorsal pancreatography. Gastroenterology. 1985; 89(5):951958. [PubMed: 4043675]

9. Sugawa C, Walt AJ, Nunez DC, Masuyama H. Pancreas divisum: is it a normal anatomic variant? Am J Surg. 1987; 153(1):62-67. [PubMed: 3799894] 
10. Bernard JP, Sahel J, Giovannini M, Sarles H. Pancreas divisum is a probable cause of acute pancreatitis: a report of 137 cases. Pancreas. 1990; 5(3):248-254. [PubMed: 2343039]

11. Liao Z, Gao R, Wang W, et al. A systematic review on endoscopic detection rate, endotherapy, and surgery for pancreas divisum. Endoscopy. 2009; 41(5):439-444. A systematic review of ERCP detection rates for PD reported a 2.9\% prevalence of PD out of 31,413 ERCPs from 17 studies. PD prevalence varied by different geographic regions, but these apparent regional differences are likely attributable to the degree that endoscopists search for PD (by examining both ductal systems), to referral bias, or to other factors. [PubMed: 19337962]

12. Ammann RW. Alcoholic chronic pancreatitis: its relation to alcoholic acute pancreatitis. Gastroenterol Clin Biol. 1996; 20(3):312-314. [PubMed: 8763072]

13. Comfort MW, Gambill EE, Baggenstoss AH. Chronic relapsing pancreatitis: a study of 29 cases without associated disease of the biliary or gastro-intestinal tract (continued). Gastroenterology. 1946; 6:376-408. [PubMed: 20985712]

14. Barthet M, Valantin V, Spinosa S, et al. Clinical course and morphological features of chronic calcifying pancreatitis associated with pancreas divisum. Eur J Gastroenterol Hepatol. 1995; 7(10): 993-998. [PubMed: 8590148]

15•. Spicak J, Poulova P, Plucnarova J, et al. Pancreas divisum does not modify the natural course of chronic pancreatitis. J Gastroenterol. 2007; 42(2):135-139. In a population with predominantly alcoholic chronic pancreatitis, groups with and without PD showed no significant differences among gender, age at onset of disease, clinical presentation, course of disease, and frequency of complications. [PubMed: 17351802]

16. Draganov P, Forsmark CE. "Idiopathic" pancreatitis. Gastroenterology. 2005; 128(3):756-763. [PubMed: 15765410]

17. Vila JJ, Vicuna M, Irisarri R, et al. Diagnostic yield and reliability of endoscopic ultrasonography in patients with idiopathic acute pancreatitis. Scand J Gastroenterol. 2010; 45(3):375-381. [PubMed: 20034361]

18. Yadav D, Lowenfels AB. Trends in the epidemiology of the first attack of acute pancreatitis: a systematic review. Pancreas. 2006; 33(4):323-330. [PubMed: 17079934]

19. Garg PK, Tandon RK, Madan K. Is biliary microlithiasis a significant cause of idiopathic recurrent acute pancreatitis? A long-term follow-up study. Clin Gastroenterol Hepatol. 2007; 5(1):75-79. [PubMed: 16931169]

20. Jacob L, Geenen JE, Catalano MF, Geenen DJ. Prevention of pancreatitis in patients with idiopathic recurrent pancreatitis: a prospective nonblinded randomized study using endoscopic stents. Endoscopy. 2001; 33(7):559-562. [PubMed: 11473324]

21. Yusoff IF, Raymond G, Sahai AV. A prospective comparison of the yield of EUS in primary vs. recurrent idiopathic acute pancreatitis. Gastrointest Endosc. 2004; 60(5):673-678. [PubMed: $15557941]$

22. Whitcomb DC, Gorry MC, Preston RA, et al. Hereditary pancreatitis is caused by a mutation in the cationic trypsinogen gene. Nat Genet. 1996; 14(2):141-145. [PubMed: 8841182]

23. Simon P, Weiss FU, Zimmer KP, et al. Spontaneous and sporadic trypsinogen mutations in idiopathic pancreatitis. JAMA. 2002; 288(17):2122. [PubMed: 12413370]

24. Cohn JA, Friedman KJ, Noone PG, et al. Relation between mutations of the cystic fibrosis gene and idiopathic pancreatitis. N Engl J Med. 1998; 339(10):653-658. [PubMed: 9725922]

25. Sharer N, Schwarz M, Malone G, et al. Mutations of the cystic fibrosis gene in patients with chronic pancreatitis. N Engl J Med. 1998; 339(10):645-652. [PubMed: 9725921]

26. Witt H, Luck W, Hennies HC, et al. Mutations in the gene encoding the serine protease inhibitor, Kazal type 1 are associated with chronic pancreatitis. Nat Genet. 2000; 25(2):213-216. [PubMed: 10835640]

27. Rossi L, Pfutzer RH, Parvin S, et al. SPINK1/PSTI mutations are associated with tropical pancreatitis in Bangladesh. A preliminary report. Pancreatology. 2001; 1(3):242-245. [PubMed: 12120202]

28. Noone PG, Zhou Z, Silverman LM, et al. Cystic fibrosis gene mutations and pancreatitis risk: relation to epithelial ion transport and trypsin inhibitor gene mutations. Gastroenterology. 2001; 121(6):1310-1319. [PubMed: 11729110] 
29. DiMagno EP. Gene mutations and idiopathic chronic pancreatitis: clinical implications and testing. Gastroenterology. 2001; 121(6):1508-1512. [PubMed: 11729132]

30. Bishop MD, Freedman SD, Zielenski J, et al. The cystic fibrosis transmembrane conductance regulator gene and ion channel function in patients with idiopathic pancreatitis. Hum Genet. 2005:1-10.

31. Choudari CP, Imperiale TF, Sherman S, et al. Risk of pancreatitis with mutation of the cystic fibrosis gene. Am J Gastroenterol. 2004; 99(7):1358-1363. [PubMed: 15233679]

32. Aoun E, Muddana V, Papachristou GI, Whitcomb DC. SPINK1 N34S is strongly associated with recurrent acute pancreatitis but is not a risk factor for the first or sentinel acute pancreatitis event. Am J Gastroenterol. 2010; 105(2):446-451. [PubMed: 19888199]

33. Steinberg WM, Chari ST, Forsmark CE, et al. Controversies in clinical pancreatology: management of acute idiopathic recurrent pancreatitis. Pancreas. 2003; 27(2):103-117. [PubMed: 12883257]

34. Pezzilli R, Morselli-Labate AM, Mantovani V, et al. Mutations of the CFTR gene in pancreatic disease. Pancreas. 2003; 27(4):332-336. [PubMed: 14576497]

35. Kopelman H, Durie P, Gaskin K, et al. Pancreatic fluid secretion and protein hyperconcentration in cystic fibrosis. New England Journal of Medicine. 1985; 312(6):329-334. [PubMed: 3969086]

36. DiMagno MJ, Lee SH, Hao Y, et al. A proinflammatory, antiapoptotic phenotype underlies the susceptibility to acute pancreatitis in cystic fibrosis transmembrane regulator $(-/-)$ mice. Gastroenterology. 2005; 129(2):665-681. [PubMed: 16083720]

37. DiMagno MJ, Lee SH, Owyang C, Zhou SY. Inhibition of acinar apoptosis occurs during acute pancreatitis in the human homologue DeltaF508 cystic fibrosis mouse. Am J Physiol Gastrointest Liver Physiol. 2010; 299(2):G400-412. [PubMed: 20522641]

38. Rosendahl J, Witt H, Szmola R, et al. Chymotrypsin C (CTRC) variants that diminish activity or secretion are associated with chronic pancreatitis. Nat Genet. 2008; 40(1):78-82. [PubMed: 18059268]

39. Felderbauer P, Hoffmann P, Einwachter H, et al. A novel mutation of the calcium sensing receptor gene is associated with chronic pancreatitis in a family with heterozygous SPINK1 mutations. BMC Gastroenterol. 2003; 3(1):34. [PubMed: 14641934]

40. Muddana V, Lamb J, Greer JB, et al. Association between calcium sensing receptor gene polymorphisms and chronic pancreatitis in a US population: role of serine protease inhibitor Kazal 1type and alcohol. World J Gastroenterol. 2008; 14(28):4486-4491. [PubMed: 18680227]

41. Groman JD, Meyer ME, Wilmott RW, et al. Variant cystic fibrosis phenotypes in the absence of CFTR mutations. N Engl J Med. 2002; 347(6):401-407. [PubMed: 12167682]

42. Knowles MR, Durie PR. What is cystic fibrosis? N Engl J Med. 2002; 347(6):439-442. [PubMed: 12167688]

43. Ko SB, Mizuno N, Yatabe Y, et al. Corticosteroids correct aberrant CFTR localization in the duct and regenerate acinar cells in autoimmune pancreatitis. Gastroenterology. 2010; 138(5):19881996. [PubMed: 20080093]

44. Gelrud A, Sheth S, Banerjee S, et al. Analysis of cystic fibrosis gener product (CFTR) function in patients with pancreas divisum and recurrent acute pancreatitis. Am J Gastroenterol. 2004; 99(8): 1557-1562. [PubMed: 15307877]

45. Garg PK, Khajuria R, Kabra M, et al. Association of SPINK1 gene mutation and CFTR gene polymorphisms in patients with pancreas divisum presenting with idiopathic pancreatitis. J Clin Gastroenterol. 2009; 43(9):848-52. [PubMed: 19593166]

46. Wilcox CM. Endoscopic therapy for pain in chronic pancreatitis: Is it time for the naysayers to throw in the towel? Gastrointest Endosc. 2005; 61(4):582-586. [PubMed: 15812412]

47. Toskes PP, Forsmark CE, DeMeo MT, et al. An open-label trial of octreotide for the pain of chronic pancreatitis. Gastroenterology. 1994; 106:A326.

48. Shiratori K, Takeuchi T, Satake K, Matsuno S. Clinical evaluation of oral administration of a cholecystokinin-A receptor antagonist (loxiglumide) to patients with acute, painful attacks of chronic pancreatitis: a multicenter dose-response study in Japan. Pancreas. 2002; 25(1):e1-5. [PubMed: 12131781] 
49. Bhardwaj P, Garg PK, Maulik SK, et al. A randomized controlled trial of antioxidant supplementation for pain relief in patients with chronic pancreatitis. Gastroenterology. 2009; 136(1):149-159. e142. [PubMed: 18952082]

50. Cahen DL, Gouma DJ, Nio Y, et al. Endoscopic versus surgical drainage of the pancreatic duct in chronic pancreatitis. N Engl J Med. 2007; 356(7):676-684. [PubMed: 17301298]

51. Cooperman AM, Siegel J, Hammerman H. Pancreas divisum--advocates and agnostics. J Clin Gastroenterol. 1989; 11(5):489-491. [PubMed: 2794426]

52. Chacko LN, Chen YK, Shah RJ. Clinical outcomes and nonendoscopic interventions after minor papilla endotherapy in patients with symptomatic pancreas divisum. Gastrointest Endosc. 2008; 68(4):667-673. The authors report that minor papilla endotherapy of patients with PD results in a clinical response or improvement in $76 \%$ with IRAP, but only $42 \%$ of those with chronic pancreatitis and $33 \%$ with chronic abdominal pain. The authors conclude that endoscopic therapy is a reasonable first step in the absence of chronic pain, but also acknowledge that short-term follow-up may be inadequate to assess response in some patients with IRAP who have long painfree intervals and/or develop chronic pain. [PubMed: 18436218]

53. Ahmed F, Sherman S. Minor papilla endotherapy in patients with symptomatic pancreas divisum. Gastrointest Endosc. 2008; 68(4):674-675. [PubMed: 18926177]

54. Delhaye M, Matos C, Arvanitakis M, Deviere J. Pancreatic ductal system obstruction and acute recurrent pancreatitis. World J Gastroenterol. 2008; 14(7):1027-1033. [PubMed: 18286683]

55. Lowes JR, Lees WR, Cotton PB. Pancreatic duct dilatation after secretin stimulation in patients with pancreas divisum. Pancreas. 1989; 4(3):371-374. [PubMed: 2660134]

56. Ng WK, Tarabain O. Pancreas divisum: a cause of idiopathic acute pancreatitis. CMAJ. 2009; 180(9):949-951. [PubMed: 19398743] 


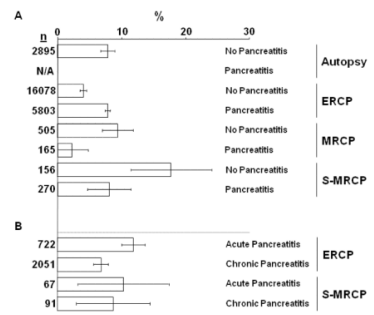

Figure 1.

(A) The prevalence of pancreas divisum (PD) without or with pancreatitis reported in autopsy, endoscopic retrograde cholangiopancreatography (ERCP), magnetic resonance cholangiopancreatography (MRCP), and secretin-MRCP (S-MRCP) studies. (B) The prevalence of PD with acute and chronic pancreatitis reported in ERCP and S-MRCP studies. Bars represent mean PD as percent of total cases (n) and error bars are $95 \%$ confidence intervals.

Figure adapted from ref [1•]. Permission from Lippincott Williams \& Wilkins. 